\title{
Multiple damage detection in unidirectional graphite-epoxy composite beams using particle swarm optimization and genetic algorithm
}

\author{
Samir Khatir*, Idir Belaidi*, Tawfiq Khatir**, Abderrachid Hamrani*, Yun-Lai Zhou***, \\ Magd Abdel Wahab***** \\ *Department Of mechanical engineering University M'hamed Bougara Boumerdes, LEMI Laboratory Research Team Modelling \\ and Simulation in Mechanical Engineering, 35000 Boumerdes, Algeria \\ **Institute of science and technology University Centre Salhi Ahmed, Naama 45000, Algeria \\ ***Department of Civil and Environmental Engineering, National University of Singapore, Engineering Drive 2, \\ Singapore 117576 \\ ****Division of Computational Mechanics, Ton Duc Thang University, Ho Chi Minh City, Vietnam \\ ****Faculty of Civil Engineering, Ton Duc Thang University, Ho Chi Minh City, Vietnam \\ ****Soete Laboratory, Faculty of Engineering and Architecture, Ghent University, Technologiepark Zwijnaarde 903, \\ B-9052 Zwijnaarde, Belgium, E-mail: magd.abdelwahab@tdt.edu.vn;magd.abdelwahab@ugent.be
}

cross $^{\text {ref }}$ http://dx.doi.org/10.5755/j01.mech.23.4.15254

\section{Introduction}

Structural damage detection and localization is a scientific field that has attracted a lot of interests in the scientific community during the recent years. There have been many studies intending to find a method to identify damage in a structure, which is also the main objective of the present study. Often, these methods are based on dynamic data analysis of the structures for damage detection and localization using optimization methods.

Numerical and experimental vibration modes were used to determine the position and the magnitude of damage using Coordinate Modal Assurance Criterion (COMAC) [1]. Messina et al. [2] have proposed an approach using natural frequency changes for the detection of damage that was later extended [3] to identify the extent of damage in several sites. Data validation was performed by free digital noise tests. This approach, however, may involve significant computational effort when it comes to large structures.

Another strategy is to use modal strain energy or mode shape to detect structural damage, which was introduced by Shi et al. [4] and Guo and Zhang [5]. The damage indicator using mode shape data to identify damage in beam-like structures has been studied [6]. Xiang et al. [7], in their work, have presented the most exciting damage detection methods using modal curvature and investigated the changes of the damage indicator between the intact and damaged state.

Genetic Algorithm (GA) has been used to solve an optimization problem formulated for the detection and identification of structural damage by Chou and Ghaboussi [8]. The damage detection in a structure using Modal Assurance Criterion (MAC), and COMAC and change in Local Frequency Ratio (LFCR) has been introduced by Khatir et al. [9].

Begambre and [10], and Gomes and Silva [1], have used two methods; one is based on the frequency sensitivity to damage and the second is based on optimization and parametric finite element modeling techniques. Gautier et al. [11] have used the inverse single damage detection and localization using model reduction based on
Proper Orthogonal Decomposition coupled by Radial Basis Functions (POD-RBF). Liu [12] investigated the location and severity of a single damaged element in a simulated planar truss by minimizing the square of the Residual Force Vector (RFV). Big Bang - Big Crunch (BB-BC) optimization method was found to be a feasible methodology to detect damage location and severity, while introducing numerous advantages compared to other methods [13].

The results found by Galvanetto and Violaris [14], in their study on a finite element model of a composite beam, showed that the developed algorithm based on proper orthogonal decomposition was capable of detecting both location and severity of damage even under variable loading condition with a high level of confidence. A vibration based Structural Health Monitoring (SHM) methods with emphasis on composite materials have been reviewed by Montalvao [15]. A new damage detection and localization technique based on the changes in vibration parameters using BAT and Particle Swarm Optimization algorithm was developed [16]. Furthermore, a damage detection and localization on thin plates based on vibration analysis using Bat algorithm was reported in [17]. The Transmissibility has been commonly used in structural health monitoring and the transmissibility coherence was put forward to system identification for extracting natural frequencies $[18,19]$ The identification of damage was formulated as an optimization problem based on Genetic Algorithm using three objective functions (change of natural frequencies, Modal Assurance Criterion MAC and MAC natural frequency).

A comparison between different techniques, was presented by Rytter [20], who proposed the following classifications:

- Level 1: Detection, i.e. to indicate qualitatively that damage might be present in the structure.

- Level 2: Localization, i.e. to provide information about the probable location of the damage.

- Level 3: Assessment, i.e. to quantify the extent of the damage.

- Level 4: Consequence, i.e. to predict the remaining life and the actual safety of the structure in a certain state of damage. 
In this paper the damage identification problem, levels 1 to 3, is addressed with Genetic Algorithm (GA) and Particle Swarm Optimization (PSO). The objective functions used in the optimization process is based on the dynamic analysis data of the structure, i.e. natural frequencies and mode shape. Various numerical examples are performed on simply supported uni-directionally reinforced graphite-epoxy beams having damage at multiple locations. The paper is organized as follows. In the next section, the methodology used in the damage detection algorithm is described. Next, in section 3, the finite element simulations of the composite beams are presented. In section 4, the optimization algorithm is described followed by the objective function in section 5 . Results and discussion are presented in section 6 , in which numerically simulated data and experimental data are used. Finally, the paper ends with a conclusion section.

\section{Methodology}

The damage detection and localization procedures, which we propose, are according to the following steps:

Modeling beam Structures using finite element method.

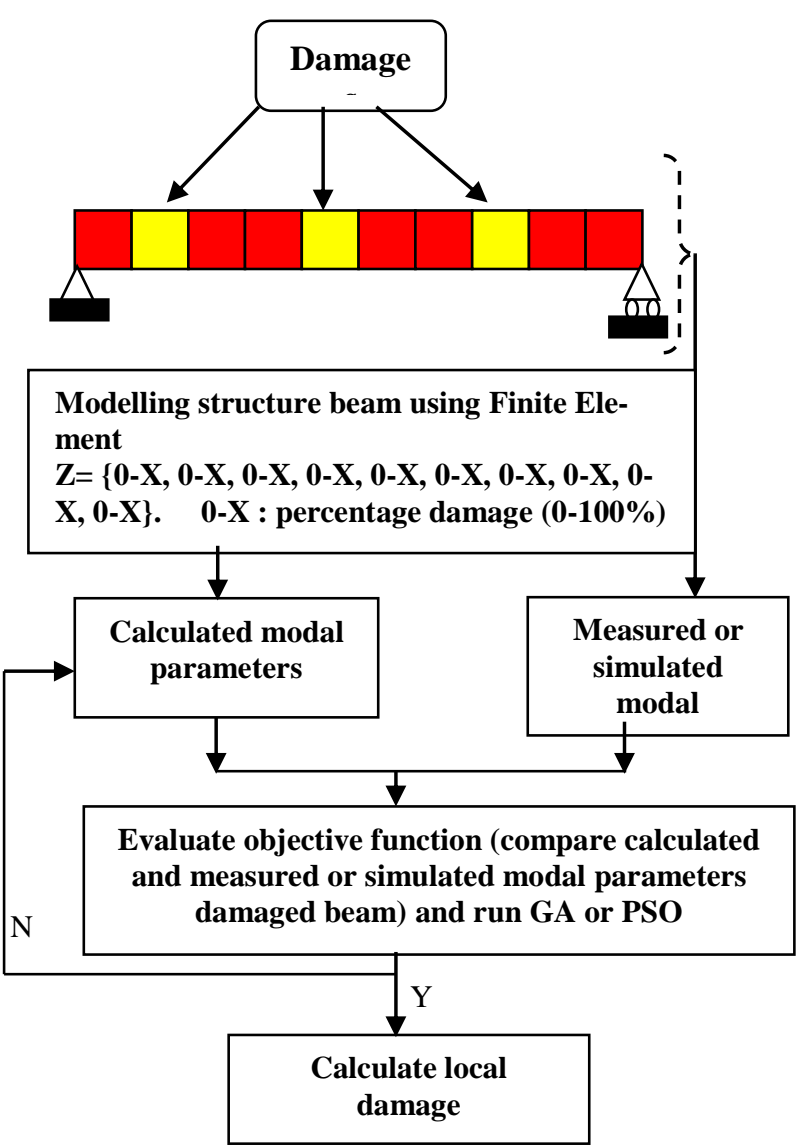

Fig. 1 Methodological approach to the damage detection and localization

Introducing damage by stiffness reduction at a global level and identifying the dynamic modal data, i.e. natural frequencies and eigenvectors, which will be used in PSO and GA. It should be noted that degradation is proportional to both longitudinal and transverse modulus. However, in finite element formulation is based on Timoshenko's Beam theory, in which a one dimensional element is considered and the effect of transverse modulus is not included.

Compare the frequencies and eigenvectors proposed by GA and PSO with frequencies and eigenvector of the structure under consideration.

Solving the inverse problem using the objective function defined in the optimization algorithm in order to locate and quantify damage using PSO or GA.

The Methodological approach to the damage detection and localization problems are illustrated in Fig. 1.

\section{Finite Element Model of composite beams}

\subsection{FE formulation}

We consider in this paper a simply supported beam of pure unidirectional composite materials of finite element SI12 as shown in Fig. 2. the derivation of the beam element is based on Timoshenko beam theory and laminated composite theory [21].

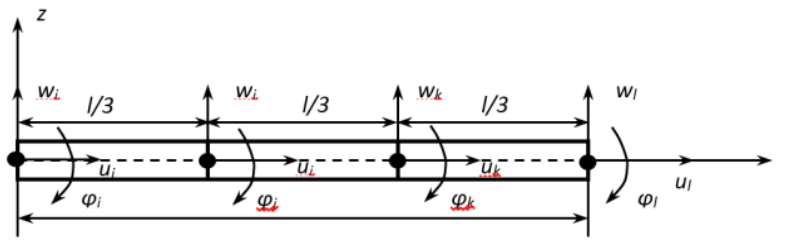

Fig. 2 Finite element SI12

We created the same beam properties, but discretized in 10 elements as shown in Figure 2. Each node of the finite element has three degrees of freedom, namely normal displacement $w$ along $z$-axis, a rotation $\gamma$ around the $y$-axis and a longitudinal displacement $u$ along the $x$ axis The shear correction coefficient is the same as for isotropic beam, i.e. $k=5 / 6$ [21].

\subsection{Experimental validation of FE model}

In order to validate our finite element approach, a carbon fiber reinforced polymer (CFRP) cantilever beam testing is considered in this study [22] (see Figure 3), where the natural frequencies are calculated and measured. The reason to choose this experiment for validation is that it has been well discussed in [28]. The CFRP cantilever beam was firstly experimented with tensile tests and then with vibration testing. During vibration tests, an impact hammer (Brüel \& Kjær) is used to excite the beam and responses are measured at different locations. Details about this experiment can refer to [22].

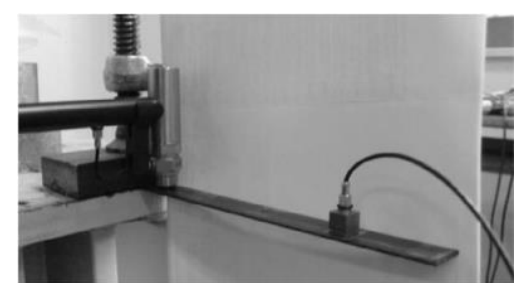

Fig. 3 Experiment setup of the CFRP beam testing [22]

The natural frequencies of this tested beam is calculated using our finite element approach and compared with the reference values as listed in Table 1, where one can find that little difference appear. This demonstrates the 
well performance of our proposed FEA approach, and thus to give confidence in further application of our model for damage detection.

\subsection{Material properties for simulated data}

The material properties used in our simulated data and beam dimensions of AS4/3501-6 graphite-epoxy [23] are given in Table 2. The composite beam is discretized in 10 elements as shown in Fig. 4. It is worth noting that in this study, we have modelled damage using a reduction in bending stiffness of the whole laminate. However, for instance if the composite beam consists of 10 plies, damage in one ply would implied a stiffness reduction of $72.9 \%\left(\frac{E b(0.9 h)^{3}}{12}=0.729 \frac{E b h^{3}}{12}\right)$.

It should be noted that we have considered herein only longitudinal bending modes in the damage detection algorithm. Furthermore, we have used unidirectional laminate, for which there is no coupling between bending modes and torsional modes. Therefore, there is no effect of shear modulus on neither the natural frequencies of bending modes nor on the damage direction algorithm.

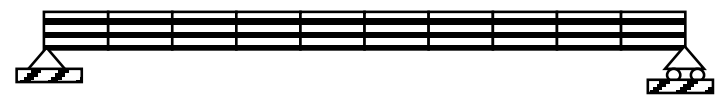

Fig. 4 Composite beam discretized in 10 elements

Table 1

Natural frequencies comparison between our FEA approach and the reference values

\begin{tabular}{|c|c|c|}
\hline Mode & Reference [28] & $\begin{array}{c}\text { Our FEA } \\
\text { model }\end{array}$ \\
\hline 1 & $67.49 \mathrm{~Hz}$ & $67.49 \mathrm{~Hz}$ \\
\hline 2 & $423.00 \mathrm{~Hz}$ & $422.89 \mathrm{~Hz}$ \\
\hline 3 & $1184.53 \mathrm{~Hz}$ & $1188.78 \mathrm{~Hz}$ \\
\hline 4 & $2321.23 \mathrm{~Hz}$ & $2338.18 \mathrm{~Hz}$ \\
\hline
\end{tabular}

Table 2

Dimension and material of composite beam

\begin{tabular}{|c|c|}
\hline Ply property & Mean value \\
\hline Length, $\mathrm{m}$ & 0.5 \\
\hline Width, $\mathrm{m}$ & 0.03 \\
\hline Thickness, $\mathrm{m}$ & 0.005 \\
\hline Longitudinal modulus, GPa & 141.96 \\
\hline Transverse Shear modulus, GPa & 6 \\
\hline Density, $\mathrm{kg} / \mathrm{m}^{3}$ & 1600 \\
\hline
\end{tabular}

\section{Genetic Algorithm and Particle Swarm Optimization}

\subsection{Genetic algorithm (GA)}

The Genetic Algorithm (GA) method is the most widely used type of Evolutionary Algorithm. A Genetic Algorithm is a probabilistic search algorithm inspired by Darwin overall survival of the fittest theory. In this optimization method, information about a problem is encoded in a gene known as an individual string (chromosome). Each of these individuals has a value of associated fitness, which is generally determined by an objective function to be minimized or maximized. Genetic algorithms have been shown to be able to solve the optimization problem by changing the operation of crossing and selection applied to individuals in the population [24]. In order to determine the ability of an individual to search a better solution, a fitness function is used to quantify how good is the solution represented by a chromosome. Depending on the problem characteristic, the fitness function can be of any form of mathematical formulation, e.g. it can be a minimized function. Mutation means a random change in the information of a chromosome and to add diversity to the genetic characteristics of the population. It is applied at a certain probability, Pm, to each gene of the offspring. The mutation probability also called mutation rate, is usually a small value, to ensure that good solutions are not distorted too much. Mutation of real variables means, that randomly created values are added to the variables selected. Fig. 5 illustrates a pseudo code of a Genetic Algorithm for damage detection.

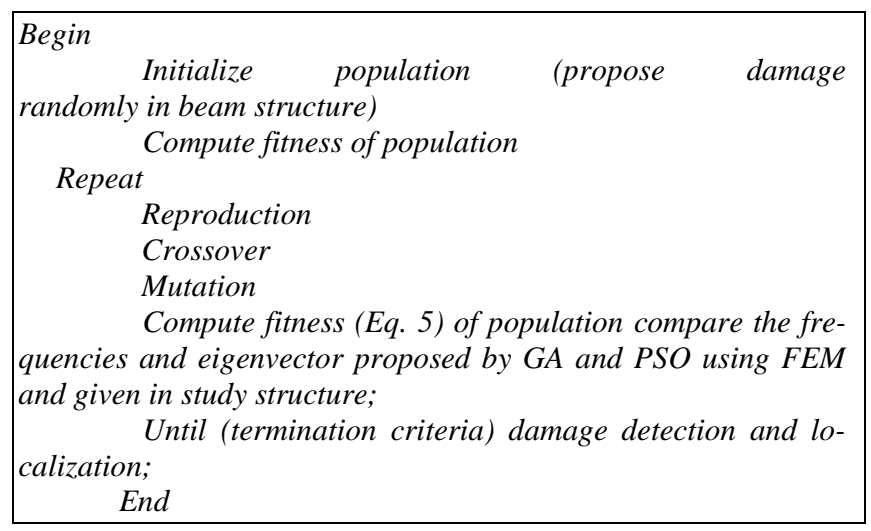

Fig. 5 Pseudo Code of GA for damage detection and localization

\subsection{Particle swarm optimization (PSO)}

The PSO algorithm was first proposed by Kennedy and Eberhart, has been used widely in the recent years and has been modified in a variety of versions that could handle the majority of optimization problems with or without the presence of constraints. It involves a swarm, modeled as a number of individual particles, moving through the search space in searching for a global optimum. The particles communicate with their neighbors over the progress made so far and adjust their moving velocity according to that information. First, a population of candidate solutions is created randomly, each of which is considered to be a particle moving through the multidimensional design space in search of the position of a global optimum. The particle can be characterized by its physical position in the space and its velocity vector, while it has the ability to remember two important information; i.e. the best position it has passed so far or a personal best (P_best) and the best position that any other particle of the swarm has passed so far or a global best (G_best). The acceleration coefficients of PSO, $c_{1}$ and $c_{2}$, represent the degree of "confidence" in the best solution found by the individual particle. The latter is possible because each particle has the ability to communicate with a number of neighboring particles, which are defined by a predetermined network topology. The 
fitness of each particle shows the quality of each solution and is evaluated by a fitness function. In every iteration the speed of the particle is updated in a stochastic way [25]. Fig. 6 illustrates a pseudo code for a particle swarm optimization PSO. The update equations for the speed and position of a particle are:

$$
\begin{aligned}
& \left\{v^{i}(t+1)\right\}=w\left\{v^{i}(t)\right\}+c_{1}\left\{r_{1}\right\}\left(\left\{x^{P b, j}\right\}-\left\{x^{j}(t)\right\}\right)+ \\
& +\left\{r_{2}\right\}\left(\left\{x^{G b}\right\}-\left\{x^{j}(t)\right\}\right), \\
& \left\{x^{i}(t+1)\right\}=\left\{x^{i}(t)\right\}+v^{i}(t+1),
\end{aligned}
$$

where $w$ is inertia weight parameter, $\left\{x^{P b, j}\right\}$ is vector of the personal best location found by the particle $j$ until current iteration, $\left\{x^{G b}\right\}$ is vector of the global best location found by the entire swarm up to the current iteration, $\left\{v^{i}(t)\right\}$ is the velocity vector of particle $j$ at time $t$, $\left\{x^{j}(t)\right\}$ is the position vector of particle $j$ at time $t, r_{1}$ and $r_{2}$ are vectors containing random numbers with uniform distribution in the interval $[0,1]$.

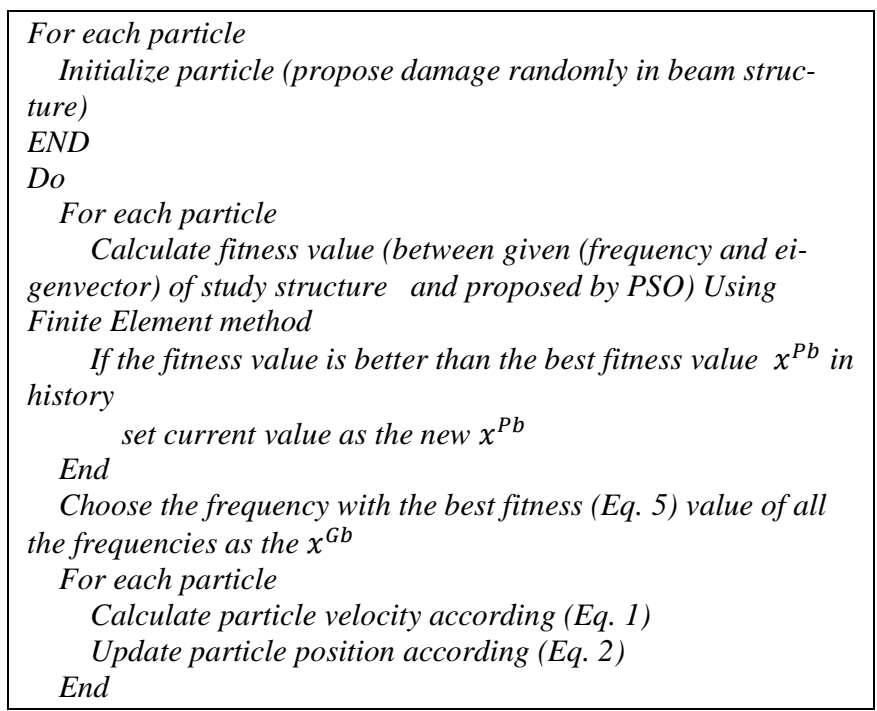

Fig. 6 Pseudo Code of PSO for damage detection and localization

\section{Objective function}

In both PSO and GA algorithms, several parameters are used to minimize the fitness function (see later Eq. (5)). In PSO, the coordinates of the particles in a twodimensional space are the parameters used to search the damage position and severity, using 100 particles. In GA, each of the 100 individuals contains two chromosomes representing the required damage parameters using a total number of iterations of 500. After several applications, a crossover coefficient of 0.8 and mutation of 0.1 were used in the GA parameters, while $c_{1}=c_{2}=2.0$ considered in the PSO method.

The objective function used in the optimization process is based on the dynamic analysis data of the structure such as natural frequencies and mode shapes. This function generates an output from the set of input variables. The objective is to modify the output in some desira- ble fashion by finding the appropriate values of input variables. In this work, the objective functions are based on Modal Assurance Criterion and changes in natural frequencies.

In our study, the Modal Assurance criterion $(M A C)$ is based on comparison between the changes in the mode shapes obtained from both damaged and undamaged tests of the studied structure. A matrix [5×5] is calculated using optimization method and FEM. MAC indicates the degree of correlation between two modes and ranges from 0 to 1 , with 1 for perfect correlation and 0 for no correlation. The deviation from 1 can be interpreted as a damage indicator of structures. The $M A C$ is defined as [26]:

$$
\operatorname{MAC}\left(\varphi_{i}, \varphi_{j}\right)=\frac{\left(\varphi_{m}^{T} \varphi_{a}\right)^{2}}{\varphi_{m}^{T} \varphi_{m} \varphi_{a}^{T} \varphi_{a}},
$$

where $\varphi_{m}^{T}$ is the measured eigenvector and $\varphi_{a}$ is the calculated eigenvector.

The natural frequencies are used as diagnostic parameters in the procedures for structural assessment using vibration monitoring. The first four natural frequencies for this numerical example are calculated. A great advantage of using natural frequencies for damage assessment in structures is that they are cheaply acquired and the approach may provide an assessment of the economic structure technique [27]:

$$
\Delta \omega=\sum_{i}^{n} \frac{\left(\omega_{i}^{m}-\omega_{i}^{a}\right)^{2}}{\left(\omega_{i}^{m}\right)^{2}},
$$

where $i$ is mode number $(i=1,2,3 \ldots n), \omega_{i}^{m}$ is measured natural frequencies and $\omega_{i}^{a}$ is calculated natural frequencies.

In this study, the statement for the objective function is defined by:

$$
\text { Fitness }=\sum_{i}^{n}\left(\omega_{i}^{m}-\omega_{i}^{a}\right)^{2}+\left|\frac{\left(\varphi_{i}^{T} \varphi_{j}\right)^{2}}{\varphi_{i}^{T} \varphi_{i} \varphi_{j}^{T} \varphi_{j}}-1\right| .
$$

\section{Results and discussion}

\subsection{Single damage}

Three different damage locations are studied, namely, damage near the hinged support (damage scenario D1), damage near the center of the beam (damage scenario D2), and damage near the roller support (damage scenario D3) positioned in the $2^{\text {nd }}, 5^{\text {th }}$ and $8^{\text {th }}$ elements with a damage rate of $60 \%$, as shown in Figs. 7, 8 and 9, respectively. A comparison of fitness evolution for the case of single damage of the three damage scenarios using both algorithms, GA and PSO, is shown in Figs. 10, 11 and 12. The comparison of damage location with GA and PSO are also given in the Tables 3 to 5 . 


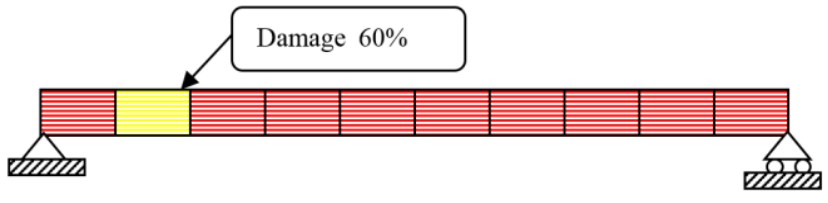

Fig. 7 Single element damage (Element 02) - damage scenario D1

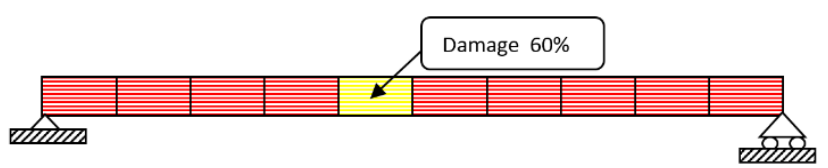

Fig. 8 Single element damage (Element 05) - damage scenario D2

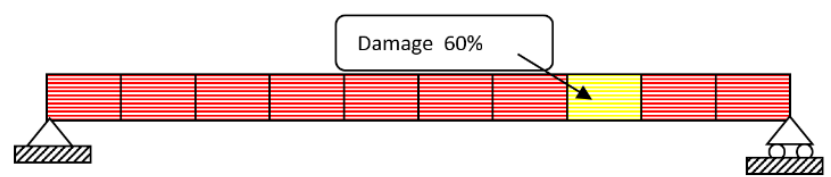

Fig. 9 Single element damage (Element eight) - damage scenario D3

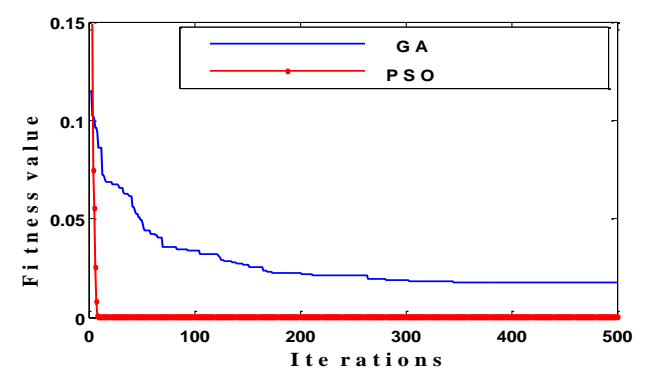

Fig. 10 Fitness convergence of PSO and GA for damage scenario D1

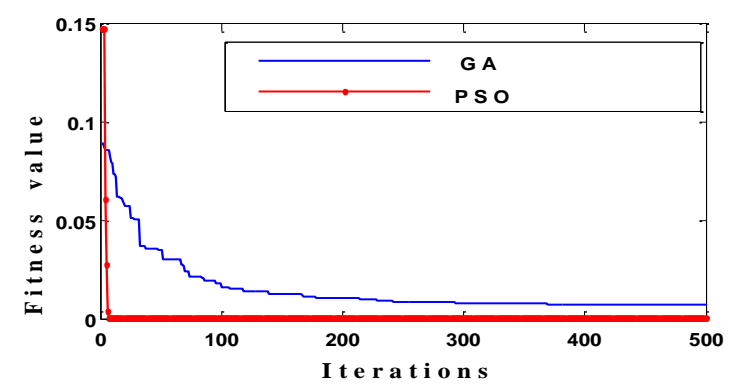

Fig. 11 Fitness convergence of PSO and GA for damage scenario D2

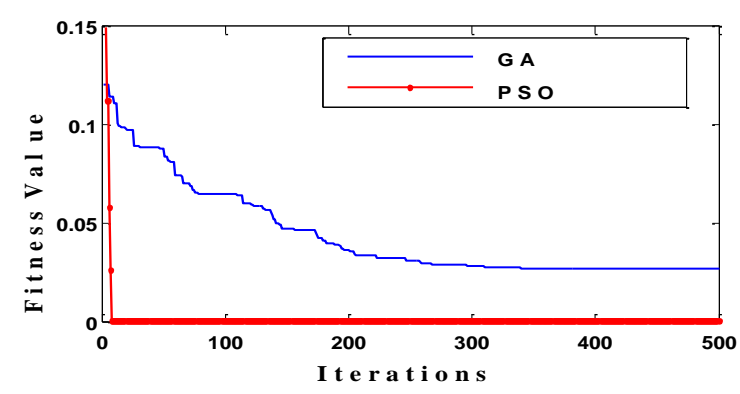

Fig. 12 Fitness convergence of PSO and GA for damage scenario D3
Table 3

Damage detection using PSO and GA for single element damage (Element 02) - damage scenario D1

\begin{tabular}{|c|c|c|c|}
\hline Elements & $\begin{array}{c}\text { Real dam- } \\
\text { age }\end{array}$ & PSO & GA \\
\hline 1 & 0 & 0 & 0.078 \\
\hline 2 & 0.6 & 0.599 & 0.485 \\
\hline 3 & 0 & 0 & 0.023 \\
\hline 4 & 0 & 0 & 0.009 \\
\hline 5 & 0 & 0 & 0.003 \\
\hline 6 & 0 & 0 & 0.059 \\
\hline 7 & 0 & 0 & 0.011 \\
\hline 8 & 0 & 0 & 0.268 \\
\hline 9 & 0 & 0 & 0.049 \\
\hline 10 & 0 & 0 & 0.244 \\
\hline
\end{tabular}

From the results of the single damage cases in the three different damage locations, it can be seen that PSO gives a more clear indication about both the position and the rate of the damage than Genetic Algorithm GA. Furthermore, it can be seen that the convergence rate that the convergence of PSO is much faster than that of GA.

Table 4

Damage detection using PSO and GA for single element damage (Element 05) - damage scenario D2

\begin{tabular}{|c|c|c|c|}
\hline Elements & Real damage & PSO & GA \\
\hline 1 & 0 & 0 & 0.065 \\
\hline 2 & 0 & 0 & 0.036 \\
\hline 3 & 0 & 0 & 0.053 \\
\hline 4 & 0 & 0 & 0.090 \\
\hline 5 & 0.6 & 0.6 & 0.495 \\
\hline 6 & 0 & 0 & 0.011 \\
\hline 7 & 0 & 0 & 0.044 \\
\hline 8 & 0 & 0 & 0.063 \\
\hline 9 & 0 & 0 & 0.053 \\
\hline 10 & 0 & 0 & 0.033 \\
\hline
\end{tabular}

Table 5

Damage detection using PSO and GA for single element damage (Element 08) - damage scenario D3

\begin{tabular}{|c|c|c|c|}
\hline Elements & Real damage & PSO & GA \\
\hline 1 & 0 & 0 & 0.015 \\
\hline 2 & 0 & 0 & 0.037 \\
\hline 3 & 0 & 0 & 0.043 \\
\hline 4 & 0 & 0 & 0.071 \\
\hline 5 & 0 & 0 & 0.015 \\
\hline 6 & 0 & 0 & 0.018 \\
\hline 7 & 0 & 0 & 0.057 \\
\hline 8 & 0.6 & 0.599 & 0.540 \\
\hline 9 & 0 & 0 & 0.041 \\
\hline 10 & 0 & 0 & 0.024 \\
\hline
\end{tabular}

\subsection{Multiple damage}

Multiple damage case is studied by considering three different damage locations (damage scenario D4), positioned at the $2^{\text {nd }}, 5^{\text {th }}$ and $8^{\text {th }}$ elements with a damage rate of $10 \%$ in element $2,30 \%$ in element 5 and $50 \%$ in element 8 as shown in Fig. 13. A comparison of fitness evolution of the three damage locations is shown in the 
Fig. 14. The comparison of three damage locations with GA and PSO is also given in Table 6.

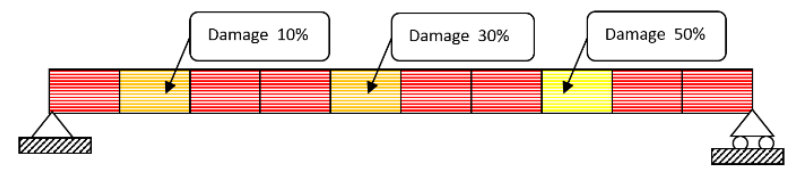

Fig. 13 Multiple element damage (Elements 2, 5 and 8) damage scenario D4

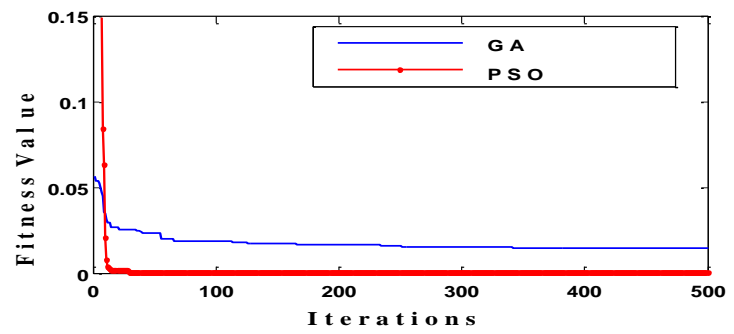

Fig. 14 Fitness convergence of PSO and GA for multiple damage - damage scenario D4

From the results of multiple damage case, it can be seen that despite several damages, PSO gives a more clear indication about both the position and the rate of the damage than GA. Furthermore, in PSO the error between real and calculated damage is very small.

Table 6 Multiple damage detection and localization using PSO and GA (Elements 2-5-8) for damage scenario D4

\begin{tabular}{|c|c|c|c|}
\hline Elements & Real damage & PSO & GA \\
\hline 1 & 0 & 0 & 0.162 \\
\hline 2 & 0.1 & 0.1 & 0.158 \\
\hline 3 & 0 & 0 & 0.007 \\
\hline 4 & 0 & 0 & 0.087 \\
\hline 5 & 0.3 & 0.299 & 0.134 \\
\hline 6 & 0 & 0 & 0.049 \\
\hline 7 & 0 & 0 & 0.025 \\
\hline 8 & 0.5 & 0.499 & 0.345 \\
\hline 9 & 0 & 0 & 0.071 \\
\hline 10 & 0 & 0 & 0.012 \\
\hline
\end{tabular}

6.3. Damage detection with noise

In order to investigate the noise effects on the results of our approach (Eq. 6). The $i^{\text {th }}$ noise response $N_{d_{i}}$ (noisy), is simulated by [28]:

$$
N_{d_{i}}(\text { Noise })=(1+\sigma \gamma) N_{d_{i}}
$$

where $\sigma$ is the noise level and $\gamma$ is a random number in the interval $[-1,1]$. In this study, $\sigma$ is considered $5 \%$ and $10 \%$. The noise levels of $5 \%$ and $10 \%$ are applied to single damage scenario (D2) and multiple damage scenario (D4, but with damage rate of $20 \%$ in element 2). Optimization results for D2 and D4 with noise levels 5\% and 10\% using PSO are shown in Tables 7 and 8, respectively.

For single and multiple damage cases with two levels of noise $5 \%$ and $10 \%$, it can be seen from Tables 7 and 8 that the results are affected. The comparison between of the predicted $d$ and real damage is still pretty accurate with small errors in some elements.

Table 7

Single damage scenario (D2) with noise levels 5\% and $10 \%$ using PSO

\begin{tabular}{|c|c|c|c|}
\hline Elements & $\begin{array}{c}\text { Real } \\
\text { damage }\end{array}$ & $\begin{array}{c}\text { Damage with } \\
\text { noise 5\% }\end{array}$ & $\begin{array}{c}\text { Damage with } \\
\text { noise } 10 \%\end{array}$ \\
\hline 1 & 0 & 0 & 0.001 \\
\hline 2 & 0 & 0 & 0 \\
\hline 3 & 0 & 0 & 0 \\
\hline 4 & 0 & 0 & 0 \\
\hline 5 & 0.5 & 0.498 & 0.491 \\
\hline 6 & 0 & 0 & 0 \\
\hline 7 & 0 & 0.001 & 0 \\
\hline 8 & 0 & 0 & 0.002 \\
\hline 9 & 0 & 0 & 0.001 \\
\hline 10 & 0 & 0 & 0 \\
\hline
\end{tabular}

Table 8 Multiple damage scenario (D4) with noise levels 5\% and $10 \%$ using PSO

\begin{tabular}{|c|c|c|c|}
\hline Elements & $\begin{array}{c}\text { Real } \\
\text { damage }\end{array}$ & $\begin{array}{c}\text { Damage with } \\
\text { noise 5\% }\end{array}$ & $\begin{array}{c}\text { Damage with } \\
\text { noise 10\% }\end{array}$ \\
\hline 1 & 0 & 0.001 & 0.010 \\
\hline 2 & 0.2 & 0.199 & 0.193 \\
\hline 3 & 0 & 0 & 0.0009 \\
\hline 4 & 0 & 0 & 0.0021 \\
\hline 5 & 0.3 & 0.297 & 0.291 \\
\hline 6 & 0 & 0 & 0.0031 \\
\hline 7 & 0 & 0 & 0.0018 \\
\hline 8 & 0.5 & 0.498 & 0.482 \\
\hline 9 & 0 & 0 & 0.001 \\
\hline 10 & 0 & 0.001 & 0 \\
\hline
\end{tabular}

\section{Conclusion}

In this paper, a method for inverse problem is proposed in order to detect and localize damage in composite beam-like structures. The proposed technique makes use of Genetic Algorithm (GA) and Particle Swarm Optimization (PSO) methods. The objective function is based on calculated natural frequencies and Modal Assurance Criterion. The results show clearly that PSO is better than GA for damage detection and localization in case of single, multiple and uniform damage scenarios. Furthermore, Genetic Algorithm has a larger computational cost than PSO. The latter exhibited in general better performance in terms of convergence speed. Noise levels were added to the modal data to test the accuracy of the method using PSO. The comparison between the predicted and real damage illustrates the efficiency of the algorithm in damage detection when noise is present in structures.

\section{References}

1. Gomes, H.; Silva, N. 2008. Some comparisons for damage detection on structures using genetic algorithms and modal sensitivity method. Applied Mathematical Modelling 32: 2216-2232.

http://dx.doi.org/10.1016/j.apm.2007.07.002.

2. Messina, A.; Jones, I.; Williams, E. 1996. Damage detection and localization using natural frequency 
changes. Proceedings of conference on Identification in Engineering Systems: 67-76.

3. Messina, A.; Williams, E.; Contursi, T. 1998. Structural damage detection by a sensitivity and statisticalbased method. Journal of Sound and Vibration 216: 791-808 http://dx.doi.org/10.1006/jsvi.1998.1728.

4. Shi, Z.; Law, S.; Zhang, L. 2000. Optimum Sensor Placement for StructuralDamage Detection. Journal of Engineering Mechanics 126: 1173-79.

http://dx.doi.org/10.1061/(ASCE)07339399(2000)126:11(1173).

5. Guo, H.; Zhang, L. 2006. A weighted balance evidence theory for structural multiple damage localization. Computer methods in applied mechanics and engineering 195: 6225-6238. http://dx.doi.org/10.1016/j.cma.2005.12.010.

6. Yazdanpanah, O.; Seyedpoor, S. 2015. A new damage detection indicator for beams based on mode shape data. Structural Engineering and Mechanics 53: 725744. http://dx.doi.org/10.12989/sem.2015.53.4.725.

7. Xiang, J.; Matsumoto, T.; Wang, Y.; Jiang, Z. 2013. Detect damages in conical shells using curvature mode shape and wavelet finite element method. International Journal of Mechanical Sciences 66: 83-93. http://dx.doi.org/10.1016/j.ijmecsci.2012.10.010.

8. Chou, J.-H.; Chaboussi, J. 2001. Genetic algorithm in structural damage detection. Computers \& structures 79: 1335-1353. http://dx.doi.org/10.1016/S0045-7949(01)00027-X.

9. Khatir, S.; Serra, R.; Belaidi, I. 2013. Détection et localisation de défauts dans des structures poutres par analyse vibratoire, 4ième Congrès Algérien de Mécanique (CAM2013) : 10 pages.

10. Begambre, O. \& Laier, J. E. 2009. A hybrid Particle Swarm Optimization-Simplex algorithm (PSOS) for structural damage identification. Advances in Engineering Software 40: 883-891.

http://dx.doi.org/10.1016/j.advengsoft.2009.01.004.

11. Khatir, S., Belaidi, I., Serra, R., Wahab, M. A., \& Khatir, T. 2016. Damage detection and localization in composite beam structures based on vibration analysis. Mechanics, 21(6): 472-479. http://dx.doi.org/10.5755/j01.mech.21.6.12526.

12. Liu, P.-L. 1995. Identification and damage detection of trusses using modal data. Journal of Structural Engineering 121: 599-608.

http://dx.doi.org/10.1061/(ASCE)07339445(1995)121:4(599).

13. Tabrizian, Z.; Afshari, E.; Amiri, G. G.; Ali Beigy, M. H.; Nejad, S. M. P. 2013. A new damage detection method: Big Bang-Big Crunch (BB-BC) algorithm. Shock and Vibration 20: 633-648. http://dx.doi.org/10.3233/SAV-130773.

14. Galvanetto, U.; Violaris, G. 2007. Numerical investigation of a new damage detection method based on proper orthogonal decomposition. Mechanical Systems and Signal Processing 21: 1346-1361. http://dx.doi.org/10.1016/j.ymssp.2005.12.007.

15. Montalvao, D.; Maia, N. M. M.; Ribeiro, A. M. R. 2006. A review of vibration-based structural health monitoring with special emphasis on composite materials, Shock and vibration digest 38: 295-326. http://dx.doi.org/10.1177/0583102406065898.

16. Khatir, S., Belaidi, I., Serra, R., Wahab, M. A., \& Khatir, T. 2016. Numerical study for single and multiple damage detection and localization in beam-like structures using BAT algorithm. Journal of Vibroengineering, 18(1): 202-213.

http://www.jve.lt/Vibro/JVE-2016-18-

1/JVE01816021884.html.

17. Khatir, A., Tehami, M., Khatir, S., \& Abdel Wahab, M. 2016. Damage detection and localization on thin plates using vibration analysis. In 23rd International Congress on Sound and Vibration (ICSV23). International Institute of Acoustics and Vibration: 8 pages.

18. Zhou, Y. L., Yang, X., \& Abdel Wahab, M. 2016. Structural health monitoring using transmissibility coherence. 5th International Conference on Fracture Fatigue and Wear 4: 200-203.

19. Khatir, S., Belaidi, I., Serra, R., Benaissa, B., \& Saada, A. A. 2015. Genetic Algorithm Based Objective Functions Comparative Study for Damage Detection and Localization in Beam Structures. In Journal of Physics: Conference Series: 628(1): 012035.

20. Rytter, A. 1993. Vibrational based inspection of civil engineering structures, $\mathrm{PhD}$ Thesis, Aalborg University, Denmark: 193 pages.

21. Rikards, R. 1991. Analysis of Laminated Structures: Course of Lectures, Riga: Riga Technical University: 114 pages.

22. Capozucca, R. 2014. Vibration of CFRP cantilever beam with damage. Composite Structures 116: 211-222. http://dx.doi.org/10.1016/j.compstruct.2014.04.027.

23. Gayathri, P.; Umesh, K.; Ganguli, R. 2010. Effect of matrix cracking and material uncertainty on composite plates. Reliability Engineering \& System Safety 95: 716-728. http://dx.doi.org/10.1016/j.ress.2010.02.004.

24. Haupt, R. L.; Haupt, S. E. 2004. Practical genetic algorithms, John Wiley \& Sons: 253 pages.

25. Plevris V.; Batavanis A.; Papadrakakis M. 2011. Optimum design of steel structures with the particle swarm optimization method based on EC3. 3rd Int. Conf. on Computational Methods in Structural Dynamics and earthquake Engineering, COMPDYN: 25 pages.

26. Ostachowicz W.; Krawczuk M.; Cartmell M. 2001. Genetic algorithms in health monitoring of structures. International Conference on Structural Control and Health Monitoring, SMART 2001, Warsaw, 22-25.

27. Vakil-Baghmisheh M.-T.; Peimani M.; Sadeghi, M. H.; Ettefagh M.M. 2008. Crack detection in beam-like structures using genetic algorithms. Applied Soft Computing 8: 1150-1160. http://dx.doi.org/10.1016/j.asoc.2007.10.003.

28. Gerist S.; Naseralavi S.; Salajegheh E. 2012. Basis pursuit based genetic algorithm for damage identification, Int J Optim Civil Eng 2: 301-19. http://en.journals.sid.ir/ViewPaper.aspx?ID=254081. 
Samir Khatir, Idir Belaidi, Tawfiq Khatir, Abdel Rachid Hamrani, Yun-Lai Zhou and Magd Abdel Wahab

\section{MULTIPLE DAMAGE DETECTION IN COMPOSITE BEAMS USING PARTICLE SWARM OPTIMIZATION AND GENETIC ALGORITHM}

S u m m a ry

This paper presents a methodology for damage detection and localization in composite beams using vibration data, Particle Swarm Optimization (PSO) and Genetic Algorithm (GA). The data was acquired by developing a program that performs dynamic analysis of unidirectional graphite-epoxy composite beams based on the Finite Element Method (FEM). The objective func- tion makes use of natural frequencies and Modal Assurance Criterion. The proposed methodology is validated using numerically simulated data and experimental data. A comparative study between the performances of PSO and GA in detecting multiple and single damage scenarios is carried out. Then, the effect of noise is investigated by taking different noise levels in the modal data. It appears that the noise has a negligible effect on the performance of the presented approaches.

Keywords: Damage detection, composite beams, modal analysis, Finite Element Analysis, Particle Swarm Optimization; Genetic Algorithm.

Received June 10, 2016 Accepted August 04, 2017 